\title{
Emergency Gastrointestinal Surgery in Patients Undergoing Antithrombotic Therapy in a Single General Hospital: a Propensity Score-matched Analysis
}

Shinya Abe ( $\sim$ sa61451689@gmail.com )

Kawakita General Hospital

Katsunori Ami

Kawakita General Hospital

Akira Katsuno

Kawakita General Hospital

Noriyasu Tamura

Kawakita General Hospital

Toshiko Harada

Kawakita General Hospital

Shunsuke Hamasaki

Kawakita General Hospital

Yusuke Kitagawa

Kawakita General Hospital

Taku Machida

Kawakita General Hospital

Naoyuki Umetani

Kawakita General Hospital

\section{Research Article}

Keywords: antithrombotic therapy, gastrointestinal surgery, emergency surgery, propensity score matched analysis

Posted Date: May 10th, 2021

DOl: https://doi.org/10.21203/rs.3.rs-80658/v2

License: (c) (1) This work is licensed under a Creative Commons Attribution 4.0 International License.

Read Full License 
Version of Record: A version of this preprint was published at BMC Gastroenterology on August 21st, 2021. See the published version at https://doi.org/10.1186/s12876-021-01897-0. 


\section{Emergency gastrointestinal surgery in patients undergoing}

\section{2 antithrombotic therapy in a single general hospital: A propensity}

\section{3 score-matched analysis}

4 Shinya Abe ${ }^{1}, K$ Katsunori Ami ${ }^{1}$, Akira Katsuno ${ }^{1}$, Noriyasu Tamura ${ }^{1}$, Toshiko Harada ${ }^{1}$, Shunsuke Hamasaki ${ }^{1}$,

5 Yusuke Kitagawa ${ }^{1}$, Taku Machida ${ }^{1}$ and Naoyuki Umetani ${ }^{1}$

6 1. Department of Digestive surgery, Kawakita General Hospital, Tokyo, Japan

7 Corresponding author: Shinya Abe, MD

8 Department of Digestive Surgery, Kawakita General Hospital,

9 1-7-3 Kitaasagaya, Suginami-ku, Tokyo, 166-0001, Japan

10 Tel: $+81-3-3119-2121$

$11 \quad$ Fax: $+81-3-3339-3604$

12 Email: sa61451689@gmail.com

13 Article category: Research article

14 Conflicts of Interest and Sources of Funding: None declared.

15 Short title: GI surgery in patients undergoing AT 


\section{ABSTRACT}

Background: This study aimed to review and evaluate the surgical outcomes, particularly

intraoperative severe blood loss and postoperative blood complications, of emergency

gastrointestinal surgery in patients undergoing antithrombotic therapy (AT). Emergency surgeries for patients with antithrombotic medication have been increasing in the aging population.

However, the effect of AT on intraoperative blood loss and perioperative complications remains

unclear.

Methods: We retrospectively reviewed 732 patients who underwent emergency gastrointestinal surgery between April 2014 and March 2019. Patients were classified into AT group and Non-AT group, and propensity score-matched analysis was performed to compare the short surgical outcomes between the groups. Additionally, risk factors in severe estimated blood loss (EBL) were assessed. The optimal value of EBL was determined using the receiver operating characteristic curve.

Results: Altogether, 64 patients received AT; 50 patients and 12, and 2 were given antiplatelet and anticoagulant, and both drugs, respectively. After propensity score matching, EBL (101 vs. 99 $\mathrm{ml} ; p=0.466)$ and postoperative complications (14 vs. 16 patients; $p=0.676$ ) were similar between 
the groups (63 patients matched paired). Intraoperative severe bleeding ( $E B L \geq 113 \mathrm{ml}$ ) occurred in 146 patients. Multivariate analysis using the full cohort revealed that anticoagulant drug use was an independent risk factor for severe bleeding (odds ratio, 6.50; 95\% CI $1.4-36 ; p=0.015$ ).

Conclusions: This study demonstrated antithrombotic drugs do not adversely affect the perioperative outcomes of emergency gastrointestinal surgery, although patients taking anticoagulant drugs or multiple antithrombotic drugs should undergo careful surgical performances and postoperative management.

Keywords: antithrombotic therapy, gastrointestinal surgery, emergency surgery, propensity score matched analysis 


\section{INTRODUCTION}

Medical science and technology improvements are leading to an increase in aging people

patients with more comorbidities. Accordingly, the number of patients taking anticoagulant drugs or antiplatelet drugs to prevent primary and secondary thromboembolism from atrial fibrillation, heart-valve replacement, and coronary-artery stenting $(1,2)$ is increasing.

In abdominal surgery, perioperative antithrombotic therapy (AT) may increase intraoperative blood loss and postoperative bleeding complications. $(3,4)$ Therefore, temporary cessation of antiplatelet and anticoagulant drugs is recommended before cardiac or non-cardiac surgery.(5-7) Hence, various perioperative AT management protocols have been developed in elective laparotomy and laparoscopic surgery, such as cholecystectomy, nephrectomy, colon resection, and rectal resection.(8-11)

Emergency general surgery patients are well-known for facing high risks for postoperative complications and death within 30 postoperative days because of an extremely high prevalence of systemic inflammatory response syndrome, sepsis, and septic shock in this subpopulation.(12-14) Although vitamin K antagonizes warfarin, most antithrombotic drugs remain in development.(15) Surgeons, therefore, are faced with difficult choices such as whether to perform emergency 
surgeries in patients undergoing AT. There is accumulating evidence on emergency surgery for patients undergoing antiplatelet therapy for acute appendicitis and acute cholecystitis.(16-18) AT may have a greater effect in patients requiring emergency surgery than in those requiring elective surgery. However, peri- and postoperative outcomes in patients receiving AT remain unclear in an emergency gastrointestinal surgery.

This study aimed to review and evaluate the surgical outcomes, particularly intraoperative severe blood loss and postoperative blood complications, of emergency gastrointestinal surgery in patients undergoing antithrombotic therapy. 


\title{
MATERIALS AND METHODS
}

\author{
Patient classification
}

We retrospectively evaluated 732 consecutive critical digestive disorder patients who underwent surgery within $24 \mathrm{~h}$ after medical reception between April 2014 and March 2019 at our institution. The operation criteria requiring emergency surgery were determined by the digestive surgeons' opinion based on the laboratory data, computed tomography findings, the abdominal findings, and patients' intention. We excluded patients who underwent surgery over $24 \mathrm{~h}$ from the hospital visit.

Patients were classified into the AT (antithrombotic therapy) group or the Non-AT group according to medication history for AT. AT is administering either or both of antiplatelet and anticoagulant drugs.

The study protocol was approved by the Ethics Committee at Kawakita General Hospital.

Treatment and postoperative management of patients undergoing antithrombotic therapy

The surgical procedure for laparotomy or laparoscopy was decided on by an individual surgeon, and we had no strict selection criteria for emergency laparoscopic surgery. During the study period, 
the percentages of emergency laparoscopic surgery in the fiscal year from 2014 to 2018 constantly increased per year at $29 \%, 48 \%, 62 \%, 63 \%$, and $75 \%$, respectively.

In the patients taking anticoagulants, unfractionated heparin was postoperatively restarted immediately after surgery if there were no postoperative complications. Oral antithrombotic agents were also immediately resumed.

\section{Data collection}

Patient data such as age, gender, postoperative diagnosis, Charlson comorbidity index, body mass index (BMI), American Society of Anesthesiologists classification of physical status (ASA - PS), and preoperative laboratory data including white blood cell, platelet counts, serum level of creatine, creactive protein (CRP), prothrombin time-international normalized ratio (PT(INR)), and activated partial thromboplastin time (APTT) were retrospectively retrieved from medical records. Postoperative complications were assessed using the Clavien-Dindo classification(19), with grade II or greater defined as significant. Data on surgical procedure, operating time, estimated blood loss, and perioperative blood transfusion were also obtained from the medical charts and records. Postoperative complications were defined as adverse events occurred in 30 days after surgery. 
Statistical analysis

Categorized variables were assessed by using the chi-squared test or Fisher's exact probability test, and continuous variables were assessed by using the Student's test or Wilcoxon test.

Propensity score matching was used to minimize the selection bias due to unbalanced baseline characteristics between patients in the AT and Non-AT groups for emergency gastrointestinal surgery.(20) The parameters that were different between both groups and other potential factors that could influence surgical outcomes such as age, gender, type of postoperative diagnosis, Charlson comorbidity index, and ASA - PS were selected to create a propensity score ranging from zero to one using a logistic regression model. Subsequently, we performed a 1:1 match between both groups, using the nearest neighbor matching with a caliper set to 0.2 of the standard deviation of the propensity score logit.(21)

To determine the optimal cut-off value for perioperative severe bleeding, a receiver operating characteristic (ROC) curve was used. Variables with $p$ value $<0.05$ on univariate analysis were further evaluated using logistic regression analyses in multivariate analyses. All statistical analyses 
were performed using JMP software ver. Pro 11.0 (SAS Institute Inc., Cary, NC, USA). $P$ values $<0.05$

were considered statistically significant. 


\section{RESULTS}

Altogether, 732 patients who underwent emergency gastrointestinal surgery for critical

digestive disorders were analyzed during the study period (median age: 53 years old, 400 male patients (55\%), mostly postoperative diagnosis: appendicitis (60\%)). 64 (8.7\%) patients were under antithrombotic therapy (AT) in the present study. We classified the patients under AT into 3 groups, such as antiplatelet (50 patients) or anticoagulant (12) or both drugs group (2), respectively. Because the risk of perioperative bleeding complications is probably different in each therapy. The patient profile on chronic antithrombotic medication is shown in Table 1 . The most common reason for the antiplatelet use was stroke followed by ischemic heart disease. In contrast, atrial fibrillation was the leading cause for anticoagulant use. The breakdown of the antiplatelets was aspirin (30 patients), clopidogrel (14), cilostazol (5), ticlopidine (2) and dipyridamole (1), whereas that of the anticoagulants was warfarin (11 patients), apixaban (1), edoxaban (1) and dabigatran (1).

The baseline characteristics of the patients in each group are summarized in Table 2. Before propensity score matching, it was observed that the AT group were older, had higher Charlson comorbidity index, poor ASA - PS, and had a higher white blood cell count than the Non-AT group. 
The predominant kinds of postoperative diagnosis of the AT and the Non-AT group are small bowel obstruction, appendicitis, and upper gastrointestinal (UGI) perforation. Therefore, we should have adjusted a large number of differences of baseline characteristics between the AT and Non-AT groups to compare the short surgical outcomes for emergency gastrointestinal surgery. To minimize the selection bias due to unbalanced baseline characteristics between patients in the AT and Non-AT groups, we used propensity score matching using the parameters such as age, gender, type of postoperative diagnosis, Charlson comorbidity index, and ASA $\cdot P S$. Sixty-three matched pairs were created from the original cohort (Table 2).

In the present study, median of the estimated blood loss with / without laparoscopic surgery or energy device were $56 \mathrm{ml}$ vs $5 \mathrm{ml}(p<0.001)$ and $24 \mathrm{ml}$ vs $5 \mathrm{ml}(p<0.001)$, respectively. Therefore, these variables also should be adjusting. Perioperative outcomes between both groups after propensity score matching are shown in Table 3 . There are no significant differences between the groups in each parameter, such as the number of laparoscopic surgeries, power device using, mean of the operating time, and estimated blood loss. The ratio of the number of blood transfusions during the perioperative period is also similar. There is no significant difference in grade II or greater postoperative morbidity in the AT and the Non-AT groups, respectively (14 [27\%] vs. 16 
[25\%] patients, $p=0.676)$. Bleeding events developed in two $(3.2 \%)$ and zero patients in the Non-

AT and the AT groups, respectively $(p=0.094)$. Regarding other adverse events, neither stroke,

embolic, nor thrombotic events occurred in the AT group patients, whereas stroke events occurred in one patient of the Non-AT group. Death within postoperative day 30 occurred in three (4.8\%) and four (6.3\%) patients in the AT group and the Non-AT group, respectively $(p=0.6969)$. There were also no significant differences between both groups in terms of postoperative stay $(p=0.409)$ and hospital death $(p=0.752)$.

We then assessed the associations between AT and intraoperative estimated blood loss (EBL), or postoperative bleeding complications. The optimal cut off value of severe intraoperative bleeding for blood transfusions was $113 \mathrm{ml}$, and the area under the curve was 0.90 (Fig. 1). Univariate and multivariate analyses for severe intraoperative bleeding and postoperative bleeding complications were performed and shown in Table 4 and Table 5, respectively. In severe intraoperative bleeding analyses, anticoagulant drug use (odds ratio 6.50, $p=0.015$ ) was an independent risk factor together with poor ASA $\cdot \mathrm{PS}$ (odds ratio $3.77, p<0.001$ ), gastrectomy (odds ratio $2.87 \times 10^{\wedge} 7, p=$ 0.003), colectomy (odds ratio 10.13, $p<0.001$ ), cholecystectomy (odds ratio 10.22, $p<0.001$ ), and laparotomy (odds ratio 4.64, $p<0.001$ ). Concerning postoperative bleeding complications analyses, 
antithrombotic drug use (odds ratio 1.08, $p=0.947$ ), anticoagulant drug use (odds ratio 2.39, $p=$ 0.584), and dual antithrombotic drug use (odds ratio 49.11, $p=0.058$ ) were not independent risk factors similar to other variables. 


\section{DISCUSSION}

Patients undergoing antithrombotic therapy (AT) have high risks of bleeding and thrombosis from their comorbidity. $(22,23)$ As those risks tend to be high when surgical intervention is required, several perioperative period protocols have been developed to prevent complications based on AT.(5-7) In contrast, the analysis of more than 400,000 surgical cases demonstrated that emergency procedures increase the risk of major postoperative morbidity and mortality.(24) Hence, the perioperative bleeding risk for patients undergoing AT may be expected to increase, making perioperative outcomes in an emergency setting a potential concern. Generally, as older people also have more comorbidities than young people, we hypothesize that perioperative complications will occur in patients with AT than those without AT.

Similar to other studies' findings $(8,10)$, the patients in this study undergoing AT were older, have higher Charlson comorbidity index, and poor ASA - PS than those who were not undergoing AT

(Table 2). Advanced age is a well-known risk factor of cardiovascular disease, such as stroke (25)

and coronary artery disease (26).

In using pre-matching data, there were no differences in operation time and estimated blood loss, whereas there were more Clavien-Dindo classifications of grade II or greater, longer 
postoperative hospital stay, and higher hospital death rate in the AT group than in the Non-AT

group (Tables 2 and 3). A higher Carlson-comorbidity index and postoperative diagnosis are negative predictors of mortality, postoperative complications, and postoperative stay in older patients.(27)

After adjusting these biases using the propensity score matching method, the estimated blood loss, perioperative blood transfusion, overall morbidity, and postoperative hospital stay were similar between the patients in both groups. Matsuoka et al. also demonstrated that antithrombotic drugs do not affect short surgical outcomes in their propensity score matching setting.(28) Additionally, Imamura et al. reported that there were no significant differences in blood loss, severe blood loss more than $100 \mathrm{ml}$, blood transfusion, and postoperative mortality for patients with or without antithrombotic therapy in emergency laparoscopic cholecystectomy.(17) These studies' findings are line with our results, which may help surgeons in deciding the strategy for critical cases with antithrombotic therapy. Therefore, antithrombotic therapy does not affect perioperative short surgical outcomes in the situation of adjusting backgrounds.

We then evaluated the risk factors for the intraoperative severe bleeding needing blood transfusions in the all cohort. Because previous studies evaluating the correlation between blood 
loss and blood transfusion obtained cut-off values for severe bleeding using ROC curve

analysis,(29) the cut-off value in this study was determined similarly, the optimal one and AUCs

were $113 \mathrm{ml}$ and 0.90 , respectively. Although $113 \mathrm{ml}$ is less than $750 \mathrm{ml}(28)$ or $1000 \mathrm{ml}(30)$ in

previous studies, our results may be sustained with the use of new surgical instrumentations and

techniques. Energy devices, including vessel-sealing devices and ultrasonically activated devices,

have improved surgical outcomes in low anterior resection, liver transection, and hysterectomy.

(31-33) These surgical techniques may be more effective in controlling intra- and postoperative

bleeding in emergency surgery in patients undergoing antithrombotic therapy. Concerning the

need for blood transfusion in cases of severe intraoperative bleeding, antithrombotic and

antiplatelet drug use were not related. However, anticoagulant drug use was more controversial in

here (Table 5). In the elective setting, two randomized clinical studies recently demonstrated that

bleeding and thrombotic events after elective non-cardiac surgery had no significant differences

between patients with or without interruption of antiplatelet agents. $(34,35)$ Concerning abdominal

surgery, several studies demonstrated the safety and feasibility of discontinued single aspirin or

clopidogrel use in gastrointestinal surgery and colorectal resection. $(30,36,37)$ These results

including ours suggested that discontinuing single antiplatelet drugs does not increase 
perioperative bleeding even in emergency surgery. Meanwhile, our results revealed that

anticoagulant drug use is one of the risk factors for severe intraoperative bleeding. The previous study reported that bleeding and mortality for emergency surgery were significantly higher than those for elective surgery in general surgical patients receiving warfarin.(38) Therefore, surgeons should pay more attention to manage the risk of intraoperative blood loss and reduce the need for blood transfusion in emergency gastrointestinal surgery in patients taking anticoagulant drugs than those taking antiplatelet drugs.

Finally, we assessed the relationship between AT and postoperative bleeding. Here, there was a higher tendency of postoperative bleeding between patients taking and not taking dual antithrombotic drugs. The overall incidence of postoperative bleeding was low, and there were no statistical differences between the groups. The previous study reported that dual antiplatelet drugs increased postoperative bleeding.(30,39) Therefore, as multiple antithrombotic drug use may increase postoperative bleeding complications in an emergency setting, careful postoperative management is necessary.

This retrospective study has several limitations. Several types of diseases were included, and the selection of surgical approach was not randomized. Antithrombotic therapy included many types 
and different generations of antiplatelet and anticoagulant agents. The total number of patients who underwent abdominal emergency surgery was relatively small in propensity score matching analyses. A comparison of the relationship between the patients with or without anticoagulant therapy in propensity score matched analysis was not performed due to their small number. Although administration of more than two anticoagulant drugs was a negative predictor for bleeding complications $(30,39)$, the present study included only two patients. 


\section{Conclusion}

This study indicated that intraoperative blood loss and postoperative complications related to emergency gastrointestinal surgery were not significantly affected by antithrombotic therapy. However, patients taking anticoagulant drugs or multiple antithrombotic drugs should undergo careful surgical performances and postoperative management.

\section{Abbreviations}

AT : antithrombotic therapy, EBL : estimated blood loss, ASA · PS : Anesthesiologists classification of physical status, ROC curve : receiver operating characteristic, AUCs : area under curves, UGI : upper gastrointestinal

\section{Declarations}

\section{Ethical Approval}

This study was conducted according to the Declaration of Helsinki, and the study protocol was

reviewed and approved by the Ethics Committee at Kawakita General Hospital.

Informed consent was performed for each patient and obtained from all patients. 
Consent for publication

Not applicable.

Availability of supporting data

Data of the current study can be available from the corresponding author if requested.

Competing interests

The authors declare that they have no competing interests.

Funding

Not applicable.

Authors' contributions

Author contribution: Shinya Abe conceptualized and designed the study, acquired, analyzed, and

interpreted the data, and drafted the article. Katsunori Ami conceptualized and designed the study, interpreted the data, and critically revised the manuscript. Akira Katsuno acquired and interpreted 
the data, and critically revised the manuscript. Noriyasu Tamura, Tosihko Harada, Shunsuke

Hamasaki, Yusuke Kitagawa, and Taku Machida acquired the data. Naoyuki Umetani conceptualized and interpreted the data, and critically revised the manuscript. All authors approved the final submission.

Acknowledgements

Not applicable.

Authors' information

${ }^{1}$ Department of Digestive Surgery, Kawakita General Hospital, Tokyo, Japan. 


\section{REFERENCES}

1. Baron TH, Kamath PS, McBane RD. Management of antithrombotic therapy in patients

undergoing invasive procedures. N Engl J Med. 2013;368:2113-2124.

2. Mozaffarian D, Benjamin E, Go A, et al. Heart disease and stroke statistics-2016 update: a report from the American Heart Association. Circulation. 2016;133:e38-360.

3. Li L, Geraghty OC, Mehta Z, et al. Study, age-specific risks, severity, time course, and outcome of bleeding on long-term antiplatelet treatment after vascular events: a population-based cohort study. Lancet. 2017;390:490-499.

4. Kono Y, Obayashi Y, Baba Y, et al. Postoperative bleeding risk after gastric endoscopic submucosal dissection during antithrombotic drug therapy. J Gastroenterol Hepatol.

2018;33: 453-460.

5. Apfelbaum JL, Nuttall GA, Connis RT, et al. Practice guidelines for perioperative blood management: an updated report by the American Society of Anesthesiologists Task Force on Perioperative Blood Management. Anesthesiology. 2015;122:241-275. 
6. Ferraris VA, Brown J, Despotis G, et al. 2011 update to the Society of Thoracic Surgeons and the Society of Cardiovascular Anesthesiologists blood conservation clinical practice guidelines. Ann Thorac Surg. 2011;91:944-982.

7. Veitch AM, Vanbiervliet G, Gershlick A, et al. Endoscopy in patients on antiplatelet or anticoagulant therapy, including direct oral anticoagulants: British Society of Gastroenterology (BSG) and European Society of Gastrointestinal Endoscopy (ESGE) guidelines. Endoscopy. 2016;48:385-402.

8. Sakamoto Y, Fujikawa T, Kawamura Y. Safety of elective laparoscopic cholecystectomy in patients with antiplatelet therapy: lessons from more than 800 operations in a single tertiary referral institution. Asian J Endosc Surg. 2020;13:33-38.

9. Kefer JC, Desai MM, Fergany A, et al. Outcomes of partial nephrectomy in patients on chronic oral anticoagulant therapy. J Urol. 2008;180:2370-2374.

10. Nozawa H, Shinagawa T, Kawai K, et al., Laparoscopic surgery in colon cancer patients treated with chronic anti-thrombotic therapy. Surg Endosc. 2018;32:3509-3516.

11. H. Nozawa, Shinagawa T, Kawai K, et al. Laparoscopic surgery in rectal cancer patients taking anti-thrombotic therapy. Minim Invasive Ther Allied Technol. 2019;1-8. 
12. Sørensen LT, Malaki A, Wille-Jørgensen P, et al., Risk factors for mortality and

postoperative complications after gastrointestinal surgery. J Gastrointest Surg.

2007;11:903-910.

13. Havens JM, Peetz A, Do W, et al., The excess morbidity and mortality of emergency general surgery. J Trauma Acute Care Surg. 2015;78:306-311.

14. Mullen MG, Michaels AD, Mehaffey JH, et al. Risk associated with complications and mortality after urgent surgery vs. elective and emergency surgery: implications for defining "quality" and reporting outcomes for urgent surgery. JAMA Surg. 2017;152: 768774.

15. Perzborn E, Roehrig S, Straub A, et al. The discovery and development of rivaroxaban, an oral, direct factor Xa inhibitor. Nat Rev Drug Discov. 2011;10:61-75.

16. Joseph B, Rawashdeh B, Aziz H, et al. An acute care surgery dilemma: emergent laparoscopic cholecystectomy in patients on aspirin therapy. Am J Surg. 2015;209:689-694.

17. Imamura $\mathrm{H}$, Minami S, Isagawa $\mathrm{Y}$, et al. The impact of antithrombotic therapy in patients undergoing emergency laparoscopic cholecystectomy for acute cholecystitis - a single center experience. Asian J Endosc Surg. 2019. 
18. Chechik O, Inabar R, Danino B, et al. Anti-platelet therapy: no association with increased blood loss in patients undergoing open or laparoscopic appendectomy. Isr Med Assoc J.

2011;13:342-344.

19. Dindo D, Demartines N, Clavien PA. Classification of surgical complications: a new proposal with evaluation in a cohort of 6336 patients and results of a survey. Ann Surg.

2004;240:205-213.

20. Rubin D, Dudoit S, van der Laan M. A method to increase the power of multiple testing procedures through sample splitting. Stat Appl Genet Mol Biol. 2006;5.

21. Austin PC. Optimal caliper widths for propensity-score matching when estimating differences in means and differences in proportions in observational studies. Pharm Stat. 2011;10:150-161.

22. Ibanez B, James S, Agewall S, et al., 2017 ESC Guidelines for the management of acute myocardial infarction in patients presenting with ST-segment elevation: the Task Force for the management of acute myocardial infarction in patients presenting with ST-segment elevation of the European Society of Cardiology (ESC). Eur Heart J 2018;39:119-177. 
23. Lip GYH, Collet JP, Haude M, et al. Management of antithrombotic therapy in AF patients presenting with ACS and/or undergoing PCI: a summary of the Joint Consensus Document of the European Heart Rhythm Association (EHRA), European Society of Cardiology Working Group on Thrombosis, European Association of Percutaneous Cardiovascular Interventions (EAPCI) and European Association of Acute Cardiac Care (ACCA) endorsed by the Heart Rhythm Society (HRS), Asia-Pacific Heart Rhythm Society (APHRS), Latin America Heart Rhythm Society (LAHRS), and Cardiac Arrhythmia Society of Southern Africa (CASSA). Eur Heart J 2018;39:2847-2850.

24. Ingraham AM, Cohen ME, Raval MV, et al. Comparison of hospital performance in emergency versus elective general surgery operations at 198 hospitals. J Am Coll Surg. 2011;212:20-28.e21.

25. Wolff V, Jouanjus E. Strokes are possible complications of cannabinoids use. Epilepsy Behav. 2017;70:355-363.

26. Tanaka $\mathrm{T}$, Seto $\mathrm{S}$, Yamamoto $\mathrm{K}$, et al. An assessment of risk factors for the complexity of coronary artery disease using the SYNTAX score. Cardiovasc Interv Ther. 2013;28:16-21. 
27. Tahiri M, Sikder T, Maimon G, et al. The impact of postoperative complications on the recovery of elderly surgical patients. Surg Endosc. 2016;30:1762-1770.

28. Matsuoka T, Kobayashi K, Lefor AK, et al. Antithrombotic drugs do not increase intraoperative blood loss in emergency gastrointestinal surgery: a single-institution propensity score analysis. World J Emerg Surg. 2019;14:63.

29. Nanashima A, Abo T, Hamasaki K, et al. Predictive parameters of intraoperative blood loss in patients who underwent pancreatectomy. Hepato-gastroenterol. 2013;60:1217-1221.

30. Fujikawa, Tanaka A, Abe T, et al. Effect of antiplatelet therapy on patients undergoing gastroenterological surgery: thromboembolic risks versus bleeding risks during its perioperative withdrawal. World J Surg. 2015;39:139-149.

31. Gotohda N, Konishi M, Takahashi S, et al. Surgical outcome of liver transection by the crush-clamping technique combined with Harmonic FOCUS ${ }^{\mathrm{Tm}}$. World J Surg. 2012;36:21562160.

32. Rossetti D, Vitale SG, Bogani G, et al. Usefulness of vessel-sealing devices for peripartum hysterectomy: a retrospective cohort study. Updates Surg. 2015;67:301-304. 
33. Manouras A, Filippakis G, Tsekouras D, et al., Sutureless open low anterior resection with total mesorectal excision for rectal cancer with the use of the electrothermal bipolar vessel sealing system. Med Sci Monit. 2007;13:CR224-230.

34. Mantz J, Samama CM, Tubach F, et al. Impact of preoperative maintenance or interruption of aspirin on thrombotic and bleeding events after elective non-cardiac surgery: the multicentre, randomized, blinded, placebo-controlled, STRATAGEM trial. BrJ Anaesth. 2011;107:899-910.

35. Oscarsson A, Gupta A, Fredrikson M, et al. To continue or discontinue aspirin in the perioperative period: a randomized, controlled clinical trial. Br J Anaesth. 2010;104:305312.

36. Jupiter DC, Fang X, Adhikari D, et al. Safety of continued clopidogrel use in the preoperative course of gastrointestinal surgery: a retrospective cohort study. Ann Surg. 2017;265:370378.

37. Yoshimoto Y, Fujikawa T, Tanaka A, et al. Optimal use of antiplatelet agents, especially aspirin, in the perioperative management of colorectal cancer patients undergoing laparoscopic colorectal resection. World J Surg Oncol. 2019;17:92. 
38. Belli S, Aytac HO, Yabanoglu H, et al. Results of surgery in general surgical patients

receiving warfarin: retrospective analysis of 61 patients. Int Surg. 2015;100:225-232.

39. Fujikawa T, Tanaka A, Abe T, et al. Does antiplatelet therapy affect outcomes of patients receiving abdominal laparoscopic surgery? Lessons from more than 1,000 laparoscopic operations in a single tertiary referral hospital. J Am Coll Surg. 2013;217:1044-1053. 
Figure Legend

Figure 1

Correlation between intraoperative estimated blood loss and perioperative blood transfusion.

Receiver operating characteristic curves predicting perioperative blood transfusion by

intraoperative estimated blood loss is represented. The area under the curve is 0.90 . The optimal cut-off value determined for intraoperative estimated blood loss is $113 \mathrm{ml}$. 
Table 1 - Patient profile on chronic antithrombotic medication

\begin{tabular}{lccc}
\hline & Antiplatelets & Anticoagulants & Both \\
\hline Patient history or current comorbidities & $(\mathrm{n}=50)$ & $(\mathrm{n}=12)$ & $(\mathrm{n}=2)$ \\
Stroke & $23(46 \%)$ & $2(17 \%)$ & 0 \\
Ischemic heart disease & $20(40 \%)$ & 0 & $1(50 \%)$ \\
Artificial fibrillation & $1(2 \%)$ & $8(67 \%)$ & $1(50 \%)$ \\
Peripheral artery disease & $1(2 \%)$ & $1(8 \%)$ & 0 \\
Cardiac valve replacement & $1(2 \%)$ & 0 & 0 \\
Deep vein thrombosis & 0 & $1(8 \%)$ & 0 \\
Central retinal artery occlusion & $1(2 \%)$ & 0 & 0 \\
Unknown & $3(6 \%)$ & 0 & 0 \\
\hline
\end{tabular}


Table 2 - Baseline characteristics of patients in the cohort

\begin{tabular}{|c|c|c|c|c|c|c|c|c|}
\hline \multirow[b]{2}{*}{ Variables } & & \multirow[b]{2}{*}{$\mathrm{n}=732$} & \multicolumn{3}{|c|}{ No-matched cohort } & \multicolumn{3}{|c|}{ Matched cohort } \\
\hline & & & AT $(n=64)$ & Non-AT $(n=668)$ & $p$ value & $\operatorname{AT}(n=63)$ & Non-AT $(\mathrm{n}=63)$ & $p$ value \\
\hline \multicolumn{9}{|l|}{ Patient-related factors } \\
\hline Age $*$ & Years & $51(34-74)$ & $83(76-8)$ & $47(32-68)$ & $<0.001$ & $83(76-87)$ & $81(69-87)$ & 0.214 \\
\hline Gender & Male & $400(55 \%)$ & $39(61 \%)$ & $361(54 \%)$ & 0.290 & $39(62 \%)$ & 32 (51\%) & 0.209 \\
\hline \multirow[t]{9}{*}{ Postoperative diagnosis } & Appendicitis & $441(60 \%)$ & $9(14 \%)$ & $432(65 \%)$ & $<0.001$ & $9(14 \%)$ & $10(16 \%)$ & \\
\hline & Small bowel obstruction & $142(19 \%)$ & $33(52 \%)$ & $109(16 \%)$ & & $33(52 \%)$ & $28(44 \%)$ & \\
\hline & UGI perforation & $47(6 \%)$ & $8(12 \%)$ & $39(6 \%)$ & & $8(13 \%)$ & $9(14 \%)$ & \\
\hline & Cholecystitis & $34(5 \%)$ & $3(5 \%)$ & $34(5 \%)$ & & $3(5 \%)$ & 0 & \\
\hline & Large bowel perforation & $30(4 \%)$ & $4(6 \%)$ & $31(4 \%)$ & & $4(6 \%)$ & $7(11 \%)$ & \\
\hline & Large bowel obstruction & $23(3 \%)$ & $2(3 \%)$ & $21(3 \%)$ & & $2(3 \%)$ & $8(13 \%)$ & \\
\hline & Bacterial peritonitis & $7(1 \%)$ & $1(2 \%)$ & $6(1 \%)$ & & $1(1 \%)$ & 0 & \\
\hline & Gastrointestinal bleeding & $6(1 \%)$ & $3(5 \%)$ & $3(0.5 \%)$ & & $3(5 \%)$ & $1(1 \%)$ & \\
\hline & Others & $2(0.2 \%)$ & $1(0.2 \%)$ & $1(0.2 \%)$ & & & & \\
\hline \multirow[t]{5}{*}{ Charlson comorbidity index } & 0 & $572(78 \%)$ & $32(50 \%)$ & $540(81 \%)$ & $<0.001$ & $32(51 \%)$ & 37 (59\%) & 0.174 \\
\hline & 1 & $95(13 \%)$ & $21(33 \%)$ & $74(11 \%)$ & & $21(33 \%)$ & $13(20 \%)$ & \\
\hline & 2 & $41(6 \%)$ & $9(14 \%)$ & $32(5 \%)$ & & $9(14 \%)$ & $8(13 \%)$ & \\
\hline & 3 & $13(2 \%)$ & $1(1 \%)$ & $12(2 \%)$ & & $1(2 \%)$ & $5(8 \%)$ & \\
\hline & $\geq 4$ & $11(2 \%)$ & $1(1 \%)$ & $10(1 \%)$ & & 0 & 0 & \\
\hline \multirow[t]{4}{*}{ ASA $\cdot$ PS } & 1 & $356(49 \%)$ & 0 & $356(53 \%)$ & $<0.001$ & 0 & $4(6 \%)$ & 0.125 \\
\hline & 2 & $306(42 \%)$ & $45(70 \%)$ & $261(39 \%)$ & & $45(71 \%)$ & $43(68 \%)$ & \\
\hline & 3 & $64(8 \%)$ & $19(30 \%)$ & $45(7 \%)$ & & $18(29 \%)$ & $16(26 \%)$ & \\
\hline & 4 & $5(1 \%)$ & 0 & $5(1 \%)$ & & 0 & 0 & \\
\hline Malignancy & yes & $29(4 \%)$ & $2(3 \%)$ & $27(4 \%)$ & 0.719 & $2(3 \%)$ & $8(13 \%)$ & 0.048 \\
\hline \multicolumn{9}{|l|}{ Preoperative laboratory data } \\
\hline White blood cell ${ }^{*}$ & $/ \mu \mathrm{L}$ & $12300(9500-15400)$ & $10500(7525-13200)$ & $12600(9600-15600)$ & $<0.001$ & $10600(7600-13300)$ & $9900(6800-14500)$ & 0.725 \\
\hline Hemoglobin* & $\mathrm{g} / \mathrm{dL}$ & $13.8(12.5-15.0)$ & $12.6(11.1-14.3)$ & $13.9(12.6-15.1)$ & $<0.001$ & $12.6(11.0-14.3)$ & $13.3(11.8-15.0)$ & 0.147 \\
\hline Platelet* & $\times 10^{4} / \mu \mathrm{l}$ & $23.0(18.8-28.1)$ & $22.0(13.1-36.1)$ & $19.5(14.0-28.9)$ & 0.455 & $21.7(13.0-36.6)$ & $19.0(13.3-33.2)$ & 0.742 \\
\hline Creatinine* & $\mathrm{U} / \mathrm{l}$ & $0.77(0.64-0.94)$ & $1.07(0.88-1.70)$ & $0.75(0.63-0.91)$ & $<0.001$ & $1.06(0.88-1.66)$ & $0.83(0.68-1.10)$ & 0.003 \\
\hline C-reactive protein ${ }^{*}$ & $\mathrm{mg} / \mathrm{dl}$ & $1.9(0.23-6.81)$ & $1.99(0.22-9.56)$ & $1.89(0.23-6.65)$ & 0.706 & $1.90(0.22-8.85)$ & $1.17(0.13-9.31)$ & 0.934 \\
\hline PT(INR) ${ }^{*}$ & & $1.09(1.02-1.19)$ & $1.13(1.06-1.36)$ & $1.09(1.02-1.17)$ & $<0.001$ & $1.13(1.06-1.35)$ & $1.08(1.01-1.16)$ & 0.005 \\
\hline APTT $^{*}$ & sec & $28.8(26.6-31.6)$ & $29.8(27.1-33.3)$ & $28.7(26.6-31.3)$ & 0.020 & $29.7(27.0-33.1)$ & $28.0(25.6-29.8)$ & 0.003 \\
\hline
\end{tabular}

AT : antithrombotic therapy, * : Median (Quartile), BMI : body mass index, ASA $\cdot$ PS : American Society of Anesthesiologists classification of physical status

PT(INR) : prothrombin time-international normalized ratio, APTT : activated partial thromboplastin time 
Table 3 - Perioperative outcomes of patients who underwent emergency gastrointestinal surgery

\begin{tabular}{|c|c|c|c|c|c|c|c|c|}
\hline \multirow[b]{2}{*}{ Variables } & & \multirow[b]{2}{*}{$\mathrm{n}=732$} & \multicolumn{3}{|c|}{ No-matched cohort } & \multicolumn{3}{|c|}{ Matched cohort } \\
\hline & & & AT $(\mathrm{n}=64)$ & Non-AT (n = 668) & $p$ value & AT $(\mathrm{n}=63)$ & Non-AT (n = 63) & $p$ value \\
\hline Laparoscopic surgery & Present & $402(55 \%)$ & $14(22 \%)$ & $338(58 \%)$ & $<0.001$ & $14(22 \%)$ & $17(27 \%)$ & 0.501 \\
\hline Energy device & Present & $395(53 \%)$ & $26(41 \%)$ & $369(55 \%)$ & 0.025 & $25(40 \%)$ & $25(40 \%)$ & 1.000 \\
\hline Operating time* & $\min$ & $94(71-124)$ & $100(62-128)$ & $93.5(71-124)$ & 0.927 & $36(10-299)$ & $53(5-251)$ & 0.955 \\
\hline Estimated blood loss* & $\mathrm{ml}$ & $10(2-70)$ & $40.5(10-307)$ & $8(2-61)$ & $<0.001$ & $101(62-129)$ & $99(74-131)$ & 0.466 \\
\hline Intra- and postoperative blood transfusion & Present & $1(0.2 \%)$ & $11(17 \%)$ & $25(4 \%)$ & $<0.001$ & $11(17 \%)$ & $7(11 \%)$ & 0.309 \\
\hline Overall complications & Present & $61(8.3 \%)$ & $21(22 \%)$ & $40(6.0 \%)$ & $<0.001$ & $14(27 \%)$ & $16(25 \%)$ & 0.676 \\
\hline Bleeding & Present & $8(1.1 \%)$ & $3(4.7 \%)$ & $5(0.75 \%)$ & 0.025 & 0 & $2(3.2 \%)$ & 0.094 \\
\hline Stroke & Present & $3(0.4 \%)$ & $3(0.5 \%)$ & 0 & 0.458 & $1(1.6 \%)$ & 0 & 0.238 \\
\hline Pulmonary & Present & $12(1.6 \%)$ & $4(6.3 \%)$ & $8(1.2 \%)$ & 0.016 & $2(3.2 \%)$ & $3(4.8 \%)$ & 0.647 \\
\hline Renal/urological & Present & $7(1.0 \%)$ & $1(1.6 \%)$ & $6(0.9 \%)$ & 0.630 & $2(3.2 \%)$ & 0 & 0.094 \\
\hline Surgical site infection & Present & $29(3.7 \%)$ & $5(7.8 \%)$ & $24(3.6 \%)$ & 0.138 & $7(11.1 \%)$ & $5(7.9 \%)$ & 0.543 \\
\hline Anastomotic failure & Present & $4(0.5 \%)$ & $1(1.6 \%)$ & $3(0.5 \%)$ & 0.335 & $2(3.2 \%)$ & $1(1.6 \%)$ & 0.555 \\
\hline Death within 30 days after surgery & Present & $17(2.3 \%)$ & $5(7.8 \%)$ & $12(1.8 \%)$ & 0.013 & $3(4.8 \%)$ & $4(6.3 \%)$ & 0.697 \\
\hline Hospital stay after surgery* & Days & $5(4-10)$ & $13(9-22)$ & $5(4-9)$ & $<0.001$ & $13(9-22)$ & $10(7-20)$ & 0.409 \\
\hline Hospital death & Present & $25(3.4 \%)$ & $6(9.4 \%)$ & $19(2.8 \%)$ & 0.020 & $5(8 \%)$ & $6(10 \%)$ & 0.752 \\
\hline
\end{tabular}

* : Median (Quartile), AT : antithrombotic therapy 
Table 4 - Univariate analysis for severe intraoperative bleeding (EBL $\geq 113 \mathrm{~mL}$ ) and postoperative bleeding complication

\begin{tabular}{|c|c|c|c|c|c|}
\hline \multirow{2}{*}{ Variables } & \multirow[b]{2}{*}{$n=732$} & \multicolumn{2}{|c|}{ Severe intraoperative bleeding (EBL $\geq 113$} & \multicolumn{2}{|c|}{ Postoperative bleeding complication } \\
\hline & & Present & $p$ value & Present & $p$ value \\
\hline \multicolumn{6}{|l|}{ Age (years) } \\
\hline$<65$ & 475 & $46(9.7 \%)$ & $<0.001$ & $1(0.2 \%)$ & 0.002 \\
\hline$\geq 65$ & 257 & $100(38.9 \%)$ & & $7(2.7 \%)$ & \\
\hline \multicolumn{6}{|l|}{ Gender } \\
\hline Female & 332 & $5616.9 \%)$ & 0.057 & $5(1.5 \%)$ & 0.327 \\
\hline Male & 400 & $90(22.5 \%)$ & & $3(0.8 \%)$ & \\
\hline \multicolumn{6}{|l|}{ BMI $\left(\mathrm{kg} / \mathrm{m}^{2}\right)$} \\
\hline$<22$ & 333 & $57(17.1 \%)$ & 0.204 & $4(1.2 \%)$ & 0.282 \\
\hline$\geq 22$ & 259 & $55(21.2 \%)$ & & $1(0.4 \%)$ & \\
\hline \multicolumn{6}{|l|}{ Charlson comorbidity index } \\
\hline $1-2$ & 708 & $131(18.5 \%)$ & $<0.001$ & $6(0.9 \%)$ & 0.001 \\
\hline $3-6$ & 24 & $15(62.5 \%)$ & & $2(8.3 \%)$ & \\
\hline \multicolumn{6}{|l|}{$\mathrm{ASA} \cdot \mathrm{PS}$} \\
\hline $1-2$ & 663 & 99 (14.9\%) & $<0.001$ & $4(0.6 \%)$ & $<0.001$ \\
\hline $3-5$ & 69 & $47(68.1 \%)$ & & $4(5.8 \%)$ & \\
\hline \multicolumn{6}{|l|}{ Antithrombotic drugs } \\
\hline Absent & 668 & $124(18.6 \%)$ & 0.003 & $5(0.8 \%)$ & 0.004 \\
\hline Present & 64 & $22(34.4 \%)$ & & $3(4.7 \%)$ & \\
\hline \multicolumn{6}{|l|}{ Antiplatelet drugs } \\
\hline Absent & 682 & $113(19.6 \%)$ & 0.344 & $6(0.9 \%)$ & 0.113 \\
\hline Present & 50 & $13(25.0 \%)$ & & $2(3.9 \%)$ & \\
\hline \multicolumn{6}{|l|}{ Anticoagulant drugs } \\
\hline Absent & 722 & $136(18.9 \%)$ & $<0.001$ & $6(0.8 \%)$ & $<0.001$ \\
\hline Present & 12 & $10(76.9 \%)$ & & $2(15.4 \%)$ & \\
\hline \multicolumn{6}{|l|}{ Dual antithrombotic drugs } \\
\hline Absent & 730 & $145(19.9 \%)$ & 0.287 & $7(1.0 \%)$ & $<0.001$ \\
\hline Present & 2 & $1(50.0 \%)$ & & $1(50.0 \%)$ & \\
\hline \multicolumn{6}{|l|}{ Malignancy } \\
\hline Absent & 700 & $130(18.6 \%)$ & $<0.001$ & $7(1.0 \%)$ & 0.258 \\
\hline Present & 32 & $16(50.0 \%)$ & & $1(3.1 \%)$ & \\
\hline \multicolumn{6}{|l|}{ Appendectomy } \\
\hline Absent & 297 & $116(39.1 \%)$ & $<0.001$ & $8(2.7 \%)$ & 0.001 \\
\hline Present & 435 & $30(6.9 \%)$ & & 0 & \\
\hline \multicolumn{6}{|l|}{ Gastrectomy } \\
\hline Absent & 728 & $142(19.5 \%)$ & $<0.001$ & $8(1.1 \%)$ & 0.833 \\
\hline Present & 4 & $4(100 \%)$ & & 0 & \\
\hline \multicolumn{6}{|l|}{ Patch repair } \\
\hline Absent & 698 & $141(20.2 \%)$ & 0.434 & 7 (1\%) & 0.288 \\
\hline Present & 34 & $5(14.7 \%)$ & & $1(2.9 \%)$ & \\
\hline Colectomy & & & & & \\
\hline Absent & 669 & 99 (14.8\%) & $<0.001$ & $6(0.9 \%)$ & 0.096 \\
\hline Present & 63 & $47(74.6 \%)$ & & $2(3.2 \%)$ & \\
\hline Intestinal surgery & & & & & \\
\hline Absent & 584 & $105(18.0 \%)$ & 0.008 & $5(0.9 \%)$ & 0.221 \\
\hline Present & 148 & $41(27.7 \%)$ & & $3(2.0 \%)$ & \\
\hline Cholecystectomy & & & & & \\
\hline Absent & 697 & 131 (18.8\% & 0.001 & 7 (1\%) & 0.304 \\
\hline Present & 35 & $15(42.9 \%)$ & & $1(2.9 \%)$ & \\
\hline Drainage operation & & & & & \\
\hline Absent & 7719 & $141(19.6 \%)$ & 0.092 & $7(1.0 \%)$ & 0.021 \\
\hline Present & 13 & $5(38.5 \%)$ & & $1(7.7 \%)$ & \\
\hline Surgical approach & & & & & \\
\hline Laparoscopic surgery & 404 & $28(6.9 \%)$ & $<0.001$ & $1(0.3 \%)$ & 0.015 \\
\hline Laparotomy & 328 & $118(36.0 \%)$ & & $7(2.1 \%)$ & \\
\hline Energy device & & & & & \\
\hline Present & 395 & $72(18.2 \%)$ & 0.209 & $3(0.8 \%)$ & 0.348 \\
\hline Absent & 337 & $74(22.0 \%)$ & & $5(1.5 \%)$ & \\
\hline
\end{tabular}


Table 5 - Multivariate analysis for severe intraoperative bleeding (EBL $\geq 113 \mathrm{~mL}$ ) and postoperative bleeding complication

\begin{tabular}{|c|c|c|c|c|c|c|c|}
\hline \multirow[b]{2}{*}{ Variable } & & \multicolumn{3}{|c|}{ Severe intraoperative bleeding (EBL $\geq 113 \mathrm{ml}$ ) } & \multicolumn{3}{|c|}{ Postoperative bleeding complication } \\
\hline & & Odds ratio & $95 \% \mathrm{CI}$ & $p$ value & Odds ratio & $95 \% \mathrm{CI}$ & $p$ value \\
\hline Age (years) & $<65$ vs. $\geq 65$ & 1.54 & $0.88-2.69$ & 0.133 & 2.76 & $0.34-75.61$ & 0.377 \\
\hline Charlson comorbidity index & $1-2$ vs. $\geq 3-6$ & 2.71 & $0.90-8.26$ & 0.075 & 5.63 & $0.69-34.42$ & 0.097 \\
\hline ASA $\cdot$ PS & $1-2$ vs. $\quad \geq 3-5$ & 3.77 & $1.91-7.58$ & $<0.001$ & 2.83 & $0.49-17.34$ & 0.238 \\
\hline Antithrombotic drugs & Absent vs. Present & 0.51 & $0.22-1.14$ & 0.102 & 1.08 & $0.05-7.83$ & 0.947 \\
\hline Anticoagulant drugs & Absent vs. Present & 6.50 & $1.43-36.86$ & 0.015 & 2.39 & $0.07-76.33$ & 0.584 \\
\hline Dual antithrombotic drugs & Absent vs. Present & & & & 49.11 & $0.86-4780$ & 0.058 \\
\hline Malignancy & Absent vs. Present & 2.18 & $0.88-5.35$ & 0.093 & & & \\
\hline Appendectomy & Absent vs. Present & 0.87 & $0.34-2.47$ & 0.791 & $2.81 \times 10^{\wedge}-7$ & $0-1.2 \times 10^{\wedge}-27$ & 0.063 \\
\hline Gastrectomy & Absent vs. Present & $2.87 \times 10^{\wedge} 7$ & 3.71 - NA & 0.003 & & & \\
\hline Patch repair & Absent vs. Present & & & & & & \\
\hline Colectomy & Absent vs. Present & 10.13 & $3.66-30.59$ & $<0.001$ & & & \\
\hline Intestinal surgery & Absent vs. Present & 1.36 & $0.55-3.65$ & 0.517 & & & \\
\hline Cholecystectomy & Absent vs. Present & 10.22 & $3.17-35.47$ & $<0.001$ & & & \\
\hline Drainage operation & Absent vs. Present & & & & 3.97 & $0.18-36.17$ & 0.315 \\
\hline Laparotomy & Absent vs. Present & 4.64 & $2.59-8.60$ & $<0.001$ & 1.24 & $0.16-25.64$ & 0.850 \\
\hline
\end{tabular}

EBL : estimated blood loss, ASA · PS : American Society of Anesthesiologists classification of physical status, CI : confidence interval 
2 Figure 1. Receiver operating curves for the optimal cut off value of severe intraoperative bleeding 
Figure 1

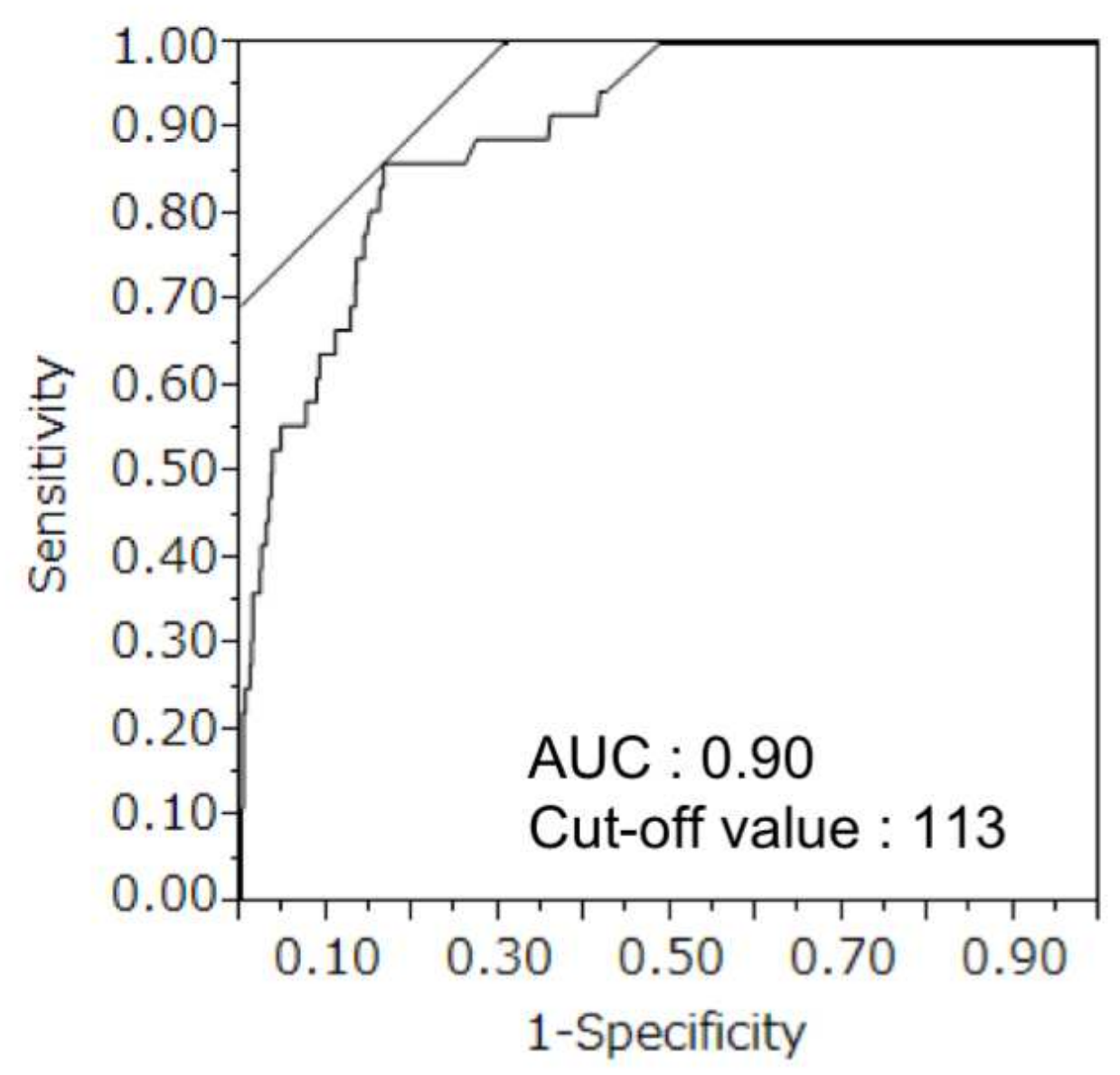




\section{Figure 1}

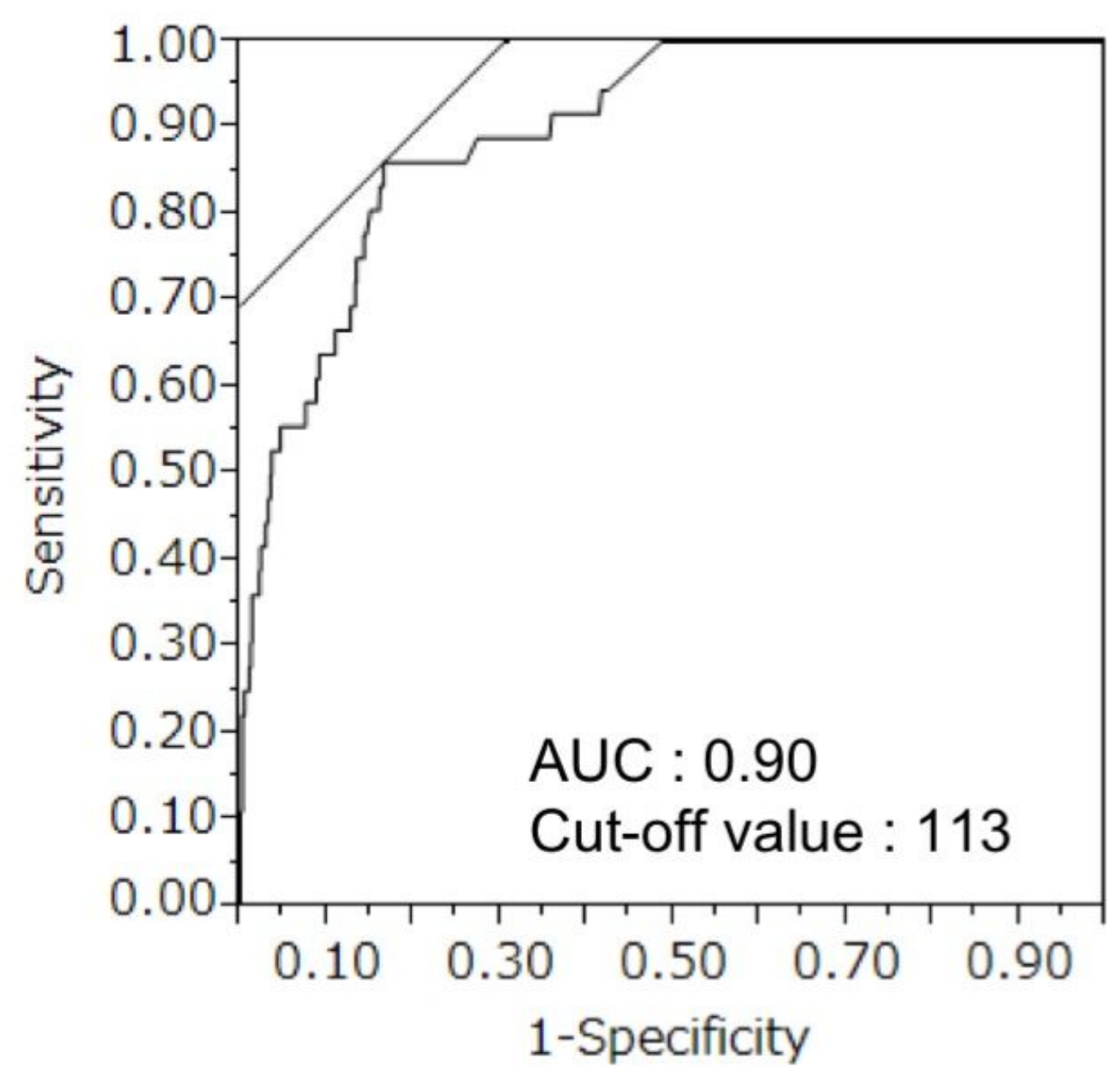

Figure 1

Receiver operating curves for the optimal cut off value of severe intraoperative bleeding. Correlation between intraoperative estimated blood loss and perioperative blood transfusion. Receiver operating characteristic curves predicting perioperative blood transfusion by intraoperative estimated blood loss is represented. The area under the curve is 0.90 . The optimal cut-off value determined for intraoperative estimated blood loss is $113 \mathrm{ml}$. 